\title{
Diagnostics of corotating interaction regions with the kinetic properties of iron ions as determined with STEREO/PLASTIC
}

\author{
P. Bochsler ${ }^{1, *}$, M. A. Lee ${ }^{1}$, R. Karrer ${ }^{2}$, L. K. Jian ${ }^{3}$, L. Ellis ${ }^{1}$, C. J. Farrugia ${ }^{1}$, A. B. Galvin ${ }^{1}$, L. M. Kistler ${ }^{1}$, \\ H. Kucharek ${ }^{1}$, E. Möbius ${ }^{1}$, M. A. Popecki ${ }^{1}$, K. D. C. Simunac ${ }^{1}$, L. M. Blush ${ }^{2}$, H. Daoudi ${ }^{2}$, P. Wurz ${ }^{2}$, B. Klecker ${ }^{4}$, \\ R. F. Wimmer-Schweingruber ${ }^{5}$, B. Thompson ${ }^{6}$, J. G. Luhmann ${ }^{7}$, C. T. Russell ${ }^{3}$, and A. Opitz ${ }^{8}$ \\ ${ }^{1}$ Space Science Center, University of New Hampshire, Durham, NH 03824, USA \\ ${ }^{2}$ Physikalisches Institut, University of Bern, 3012 Bern, Switzerland \\ ${ }^{3}$ Institute of Geophysics and Planetary Physics, UCLA, Los Angeles, CA 90024, USA \\ ${ }^{4}$ Max-Planck-Institut für extraterrestrische Physik, 85741 Garching, Germany \\ ${ }^{5}$ Institute for Experimental and Applied Physics, Christian-Albrechts-University, Leibnizstr. 11, 24098 Kiel, Germany \\ ${ }^{6}$ National Aeronautics and Space Administration, Goddard Space Flight Center, Greenbelt, MD 20771 USA \\ ${ }^{7}$ Space Science Laboratory, University of California, Berkeley, CA 94720, USA \\ ${ }^{8}$ Centre d'Etude Spatiale des Rayonnements (CNRS-UPS), 31028 Toulouse, France \\ *permanent address: Bahnhofstrasse 54, 3127 Mühlethurnen, Switzerland
}

Received: 29 May 2009 - Revised: 10 December 2009 - Accepted: 16 December 2009 - Published: 9 February 2010

\begin{abstract}
STEREO/PLASTIC determines threedimensional distributions of solar wind iron ions with unprecedented time resolution. Typically 300 to 1000 counts are registered within each $5 \mathrm{~min}$ time interval. For the present study we use the information contained in these distributions to characterize CIRs (Corotating Interaction Regions) in two test cases. We perform a consistency test for both the derived physical parameters and for the analytical model of CIRs of Lee (2000). At $1 \mathrm{AU}$ we find that apart from compositional changes the most indicative parameter for marking the time when a CIR passes a spacecraft is the angular deflection of the flow vector of particles. Changes in particle densities and the changes in magnitudes of speeds are apparently less reliable indicators of stream interfaces.
\end{abstract}

Keywords. Interplanetary physics (Discontinuities; Interplanetary shocks; Solar wind plasma)

\section{Introduction}

Corotating Interaction Regions (CIRs) have been a subject of continued interest since shortly after the discovery of the solar wind and of its two distinct states, i.e., slow and fast wind that interact in interplanetary space due to the rotation

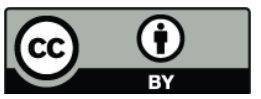

Correspondence to: $\mathrm{P}$. Bochsler (bochsler@soho.unibe.ch) of the Sun (Neugebauer and Snyder, 1966). Stream interfaces are characterized by a relatively sharp density drop and temperature rise, and an increase in the bulk velocity. It has not been clearly identified, where and how these interfaces originate near the Sun (e.g., Burlaga, 1995, or Forsyth and Marsch, 1999). Forsyth and Marsch (1999) argue that low in the corona the transition from slow to fast wind occurs on scales of a supergranule, i.e., within a few $10 \mathrm{Mm}$. Aellig (1997, 1998), and Wimmer-Schweingruber et al. (1997) used strong compositional discontinuities to show that sharp transitions from one solar wind source to a neighboring source survive undisturbed out to $1 \mathrm{AU}$. The change in the kinetic properties of the ions across such an interface is more complex because these properties are gradually modified with the radial propagation of the interface outward into space. With increasing distance from the Sun the interface changes its orientation from radial to tangential, and consequently the interaction of two adjacent streams changes from pure shearing to compression. Compression waves in the forward direction into the slow wind, and in the reverse direction into the fast wind regime, build up gradually. These compression waves steepen and eventually form a shock pair in the forward and in the reverse direction. Increasingly sophisticated models of stream interfaces have been developed over the past decades, beginning from purely hydrodynamic models (Carovillano and Siscoe, 1969; Hundhausen, 1973; Pizzo, 1978) to models including the full set of MHD-equations in three dimensions (Pizzo, 1991). In about three quarters of cases, shocks are

Published by Copernicus Publications on behalf of the European Geosciences Union. 
formed outside $1 \mathrm{AU}$, when the angle of the Parker spiral has become sufficiently oblique relative to the radial flow direction. One of the most sensitive indicators of an approaching stream interface in the ambient solar wind is the deflection of particle flows from the radial direction. Lee (2000) presented an easy-to-use analytical model of stream interfaces, describing the interrelation between various observables in a concise manner.

The PLasma And Supra-Thermal Ion Composition (PLASTIC) experiment onboard the STEREO spacecraft, with its ability to disentangle the three-dimensional velocity distribution of test particles, offers the opportunity for a renewed investigation of the effect of CIRs on test particles. We use the analytical model of Lee (2000), which offers a comparatively simple method to test the internal consistency of the data, and the consistency of the model with our data. Thus the purpose of this study is twofold: First, we test the applicability of the simplifications in the theoretical description of CIRs by Lee (2000). Second, the angular resolution of STEREO/PLASTIC has been successfully calibrated in the laboratory (cf. Karrer, 2007, and Galvin et al., 2008) and inflight using several roll-maneuvers of the spacecraft, and the result of these operations needs to be verified. Using the example of two CIRs observed with STEREO/A in May 2007, we determine the orientation of the interface at the source surface of the solar wind.

\section{The model of Lee (2000)}

The model of Lee (2000) adopts a locally planar approximation of the interface and is fully described with the components of the normal vector to the interface:

$\mathbf{n}=\frac{1}{\sqrt{\left(1+d^{2}+\left(\frac{d \Omega r}{V}\right)^{2}\right)}}\left(\frac{d \Omega r}{V} \mathbf{e}_{\mathbf{r}}, d \mathbf{e}_{\phi}, \mathbf{e}_{\theta}\right)$.

Here $\mathbf{n}$ denotes the vector normal to the interface, the $\mathbf{e}_{\mathbf{i}} \mathbf{s}$ are the unit vectors in spherical coordinates, $\Omega$ is the angular speed of the Sun, $V$ is the speed of the slow solar wind preceding the interface, and $d$ specifies the orientation of the interface at the source surface of the solar wind. Specifically if the interface is given by its heliolatitude as a function of heliolongitude, $d$ is the derivative of that function. The condition $d>0$ implies that the fast wind is $\mathrm{N}$ and $\mathrm{E}$ of the slow wind, whereas $d<0$ implies that the fast wind is $\mathrm{S}$ and E of the slow wind. The model of Lee (2000) then distinguishes four different regimes: Regime 1 is the undisturbed fast wind, which flows with a radial speed $V+\Delta V$, while Regime 4 is the undisturbed slow wind ahead of the interface, which flows with a radial speed $V$. Regime 2 follows the interface and precedes the reverse shock (or reverse pressure wave), which expands into the fast wind. Depending on the parameter $d\left(=n_{\phi} / n_{\theta}\right)$, fast particles streaming in this regime have experienced a deflection in the WE-direction and in the NS-direction. Similarly, in Regime 3 which follows Regime 4 and which lies between the forward shock (or pressure wave) and the interface, particles are deflected in the opposite direction from Regime 3. The velocity vectors, and hence the deflection angles, in Regimes 2 and 3 in Lee's model can be fully determined from kinematic considerations: Components parallel to a front are conserved across the front, while the change of the radial component can be inferred from momentum conservation in the rest frame of the slow wind. Large tilt angles (large values of $d$ ) predominantly produce WE-deflections, whereas with small tilt angles of the interface, NS-deflections dominate. The model of Lee (2000) considers the bulk flow of the plasma, it does not consider different species individually, and it does not distinguish between minor species, which often travel with flow vectors different from those of protons due to the action of wave acceleration. The action of waves, which are generated near shocks and compression regions, and which undoubtedly work differently on minor species than on protons, are not discussed in this model. Although it is evident that the analysis of the kinetic properties of protons and minor ions would yield different results, such a comparison is beyond the scope of the present paper.

\section{Observations}

Figure 1 shows the WE-flow-angles of iron ions in red and the $\mathrm{SN}$-flow angles in blue in the instrument frame as observed with the PLASTIC instrument (Galvin et al., 2008) on STEREO/A. Iron ions are well separated from all lighter ions on the basis of time-of-flight and energy classifications. Typically 300 to 1000 counts are registered within a time interval of five minutes. Flow angles have been derived with two methods. Originally, an eight-parameter-fit assuming a bi-maxwellian velocity distribution was used; one parameter was used to characterize the density, and three free parameters defined a bulk flow vector including the two angles relative to the radial direction. The remaining four parameters are given by $T_{\|}$, the two angles specifying the orientation of the $T_{\|}$symmetry axis with respect to the radial direction, and $T_{\perp}$. During the numerical analysis of larger datasets it turned out that this method sometimes converged very slowly, and consequently, the number of free parameters was reduced to six: The flow angles were henceforth determined using a moment method, i.e., using the averages of $v_{y}$, and $v_{z}$ in the instrument frame from the counts registered within the given time interval. The results of the moment method for determining the flow angles were generally consistent with the results obtained from the eight-parameter method. The data points in Figs. 1 and 2 show that the procedure produces relatively little scatter and yields reproducible results under stable conditions. Note that each data point was determined individually and independently of any other data point, and that no smoothing has been applied. The selected period in 


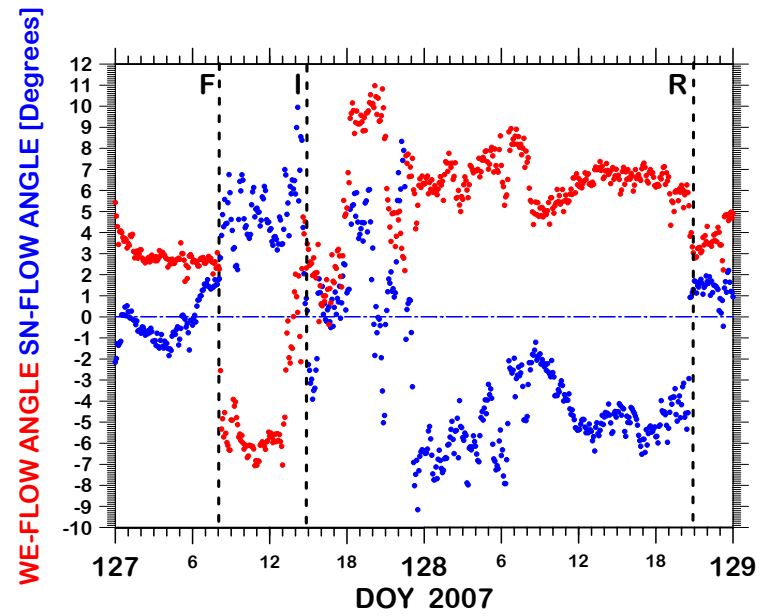

Fig. 1. Flow angles of iron ions in the vicinity of the CIR of $7 / 8$ May 2007 measured with PLASTIC on STEREO/A. The WE-flow angles (red) have not been corrected for aberration effects. The WEaberration, due to the motion of the spacecraft, is assumed to be $3^{\circ}$ as seen in the undisturbed slow wind early in DOY 127. The SNdeflection angles are shown as blue dots.

Fig. 1 shows unambiguously the discontinuities in the deflection angles near $8 \mathrm{~h} \mathrm{UT}$ on DOY 127 and at $21 \mathrm{~h} \mathrm{UT}$ of DOY 128,2007 . These discontinuities are readily associated with the forward shock (indicated by the dashed line "F") and the reverse shock "R". The line "I" indicates the time ( $\sim 15$ UT, DOY 127) when STEREO/A crossed the stream interface; it was identified according to the criteria proposed by Burlaga (1995). It roughly coincides with the flip of the flow directions from east-west to west-east.

Our second example (see Fig. 2) was observed eleven days later, also with PLASTIC on STEREO/A during the CIR of 18/19 May 2007. In this case no shock was found. Nevertheless, a deflection in WE-flow-angles as well as in $\mathrm{SN}$-flow angles of iron ions was clearly visible in the forward pressure wave, albeit the transition was somewhat smoother than in our first CIR.

\section{Methods}

\subsection{Ordering parameters}

The spiral angle of the interface within the ecliptic is determined through the interface speed, which is intermediate between the slow and fast speeds and is given by Eq. (38) in Lee (2000). The Eqs. (41-44) of Lee (2000) can then be used to calculate the EW- and $\mathrm{SN}$-deflection angles for a given slope $d$. As in Lee (2000), we use the symbol $\chi$ for the EWdeflection and $\psi$ for the SN-deflection. Subscript 3 denotes Region 3, whereas subscript 2 is for Region 2, which follows the interface before the reverse shock (or reverse pressure

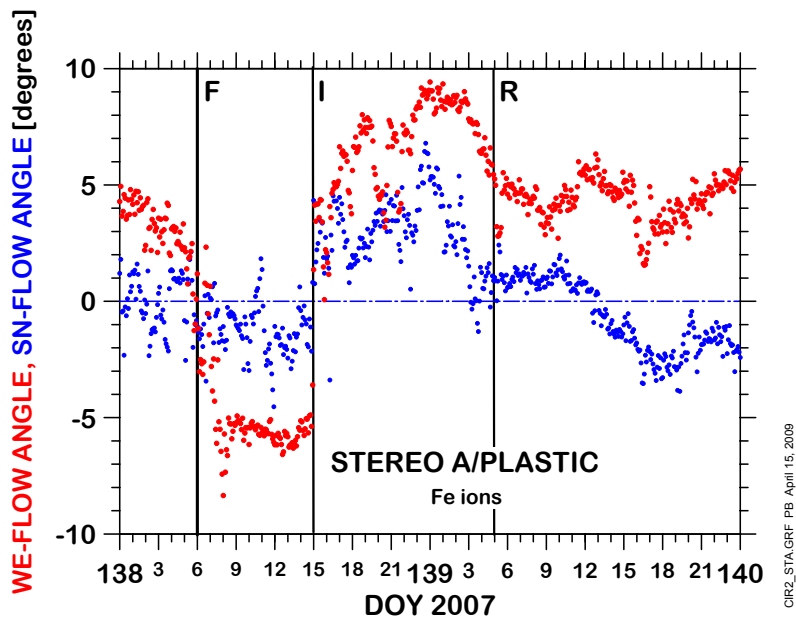

Fig. 2. (Same format as Fig. 1) Flow angles of iron ions near the CIR of 18/19 May 2007. The WE-aberration has not been corrected. This CIR has not yet developed shocks at the site of the spacecraft near 1 AU. Correspondingly, the transitions near the forward and reverse pressure waves, " $F$ " and " $R$ ", are smoother than in the example shown in Fig. 1.

wave). The slope of the interface can, in principle, be derived by dividing Lee's Eqs. (41) by (42), or by dividing (43) by (44). In both cases one finds:

$$
\frac{\tan \chi_{i}}{\tan \psi_{i}}=-\frac{\left(\mathbf{n} \cdot \mathbf{e}_{\phi}\right)}{\left(\mathbf{n} \cdot \mathbf{e}_{\theta}\right)}=-d \text {. }
$$

This result is also given in the paper by Lee (2000) in Eq. (25) for observations within the ecliptic. As we shall see in Sect. 5 this method of determining the slope of the interface works in our second case, but somewhat less satisfactorily in the first case which involves a shock. Furthermore, this approach does not include the observations of the radial speed components in the regions before and after the interface. In the following, and as an alternative, we will use a least-squarescriterion to determine the parameter $d$. This method involves all observed parameters.

\subsection{Least squares fit}

We have six observables characterizing the flow vectors between the forward and backward pressure waves (or shocks). These are in the notation of Lee (2000) $\chi_{2}$ and $\chi_{3}$, the EWdeflection angles before and after the interface, $\psi_{2}$ and $\psi_{3}$, the corresponding SN-deflection angles, and $w_{2}$ and $w_{3}$, the magnitudes of the bulk speeds of iron ions in the inertial frame. The only unknown is the parameter $d$; once $d$ has been determined, values for the observables can be calculated from the expressions given in Lee (2000), to which we assign the subscript "calc". All observables, here denoted by $\mathrm{X}$, are determined with some uncertainties $\Delta X$. The quantitity to minimize is given by 

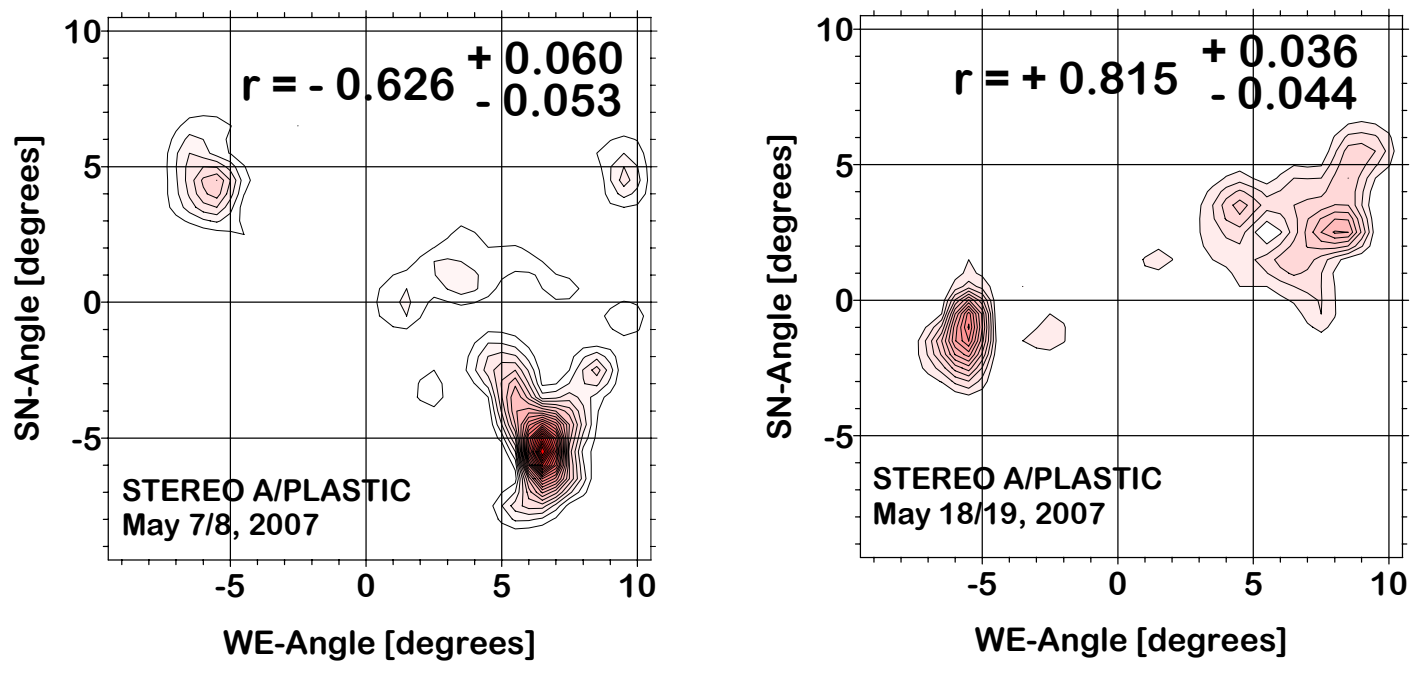

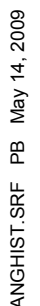

Fig. 3. Correlograms of deflection angles between the forward and reverse shocks of the CIR of 7/8 May 2007 (left panel), and between the forward and reverse pressure waves of the CIR of 18/19 May 2007 (right panel).

$$
\begin{aligned}
F & =\left(\frac{\chi_{2}-\chi_{2, \text { calc }}}{\Delta \chi_{2}}\right)^{2}+\left(\frac{\chi_{3}-\chi_{3, \text { calc }}}{\Delta \chi_{3}}\right)^{2} \\
& +\left(\frac{\psi_{2}-\psi_{2, \text { calc }}}{\Delta \psi_{2}}\right)^{2}+\left(\frac{\psi_{3}-\psi_{3, \text { calc }}}{\Delta \psi_{3}}\right)^{2} \\
& +\left(\frac{w_{2}-w_{2, \text { calc }}}{\Delta w_{2}}\right)^{2}+\left(\frac{w_{3}-w_{3, \text { calc }}}{\Delta w_{3}}\right)^{2} .
\end{aligned}
$$

The method then consists in finding a solution to the equation $\frac{\partial F}{\partial d}=0$. It differs from the method, which uses the correlation between deflection angles, as expressed in Eq. (1), since one also attempts to minimize the differences between the observed and the calculated quantities $w_{i}$, which are the magnitudes of the bulk speeds measured in the inertial frame in Regions 2 and 3. The parameter $d$ does not appear explicitly in Eq. (2); however as mentioned above, all the calculated quantities depend on $d$, following expressions given in Lee (2000).

\section{Results}

We determined the parameter $d$ using both methods outlined above. From the correlation of the deflection angles as shown in Fig. 3 one obtains $d=-1.640 \pm 0.004$ for the CIR of $7 / 8$ May 2007, whereas for the second case of 18/19 May 2007, one finds $d=+2.972 \pm 0.008$. The correlation between the deflection angles is determined by the two clusters in Fig. 3 representing the regimes before and after the interface. Note that the EW-angles have not been corrected for aberration effects; this has, however, no effect on the derivation of $d$. Note also that the given uncertainties are only the statistical uncertainties of the correlation, which are very small due to the large number of data points. It is somewhat surprising that the second case with the steeper slope of the interface is not associated with shocks, whereas in the first configuration clear shocks were observed at 1 AU. In both cases considered here the difference in speed between slow and fast solar wind was very similar and amounted to $350 \mathrm{~km} / \mathrm{s}$. One reason for the absence of a shock in the second CIR might be that the ambient temperature in the low-speed wind regime preceding the interface was about three times higher than the temperature preceding the interface of 7/8 May; therefore a shock had not yet developed at the site of the observer.

The least squares method yielded $d=-0.93$ for the first case and $d=+5.40$ for the second case. The comparison of these numbers with the results obtained from the correlation method shows the limits of these methods. The difference in determinations of $\mathrm{d}$ for Case 2 is, however, not as dramatic if expressed as tilt angles: $\tan ^{-1}(2.97)=71.4^{\circ}$ vs. $\tan ^{-1}(5.4)=79.5^{\circ}$. For the least-squares method we used uncertainties of the fluctuating deflection angles, as is evident from Figs. 1 and 2, of typically 5 degrees. We estimate realistic uncertainties of the values of $d$ obtained with this procedure to be typically $30 \%$. This one and only free parameter $d$, characterizing the shape of the interface at the source surface, is crucial for the development of a CIR out to $1 \mathrm{AU}$ and beyond.

Finally, we have compared our evaluations for the slope of the interface line at the source surface, with the shape of the neutral line in the pictures of coronal hole models of GONG (http://gong.nso.edu). At the time of the observations, the position of STEREO/A differed only a few degrees from the position of the Earth in heliospheric longitude; 

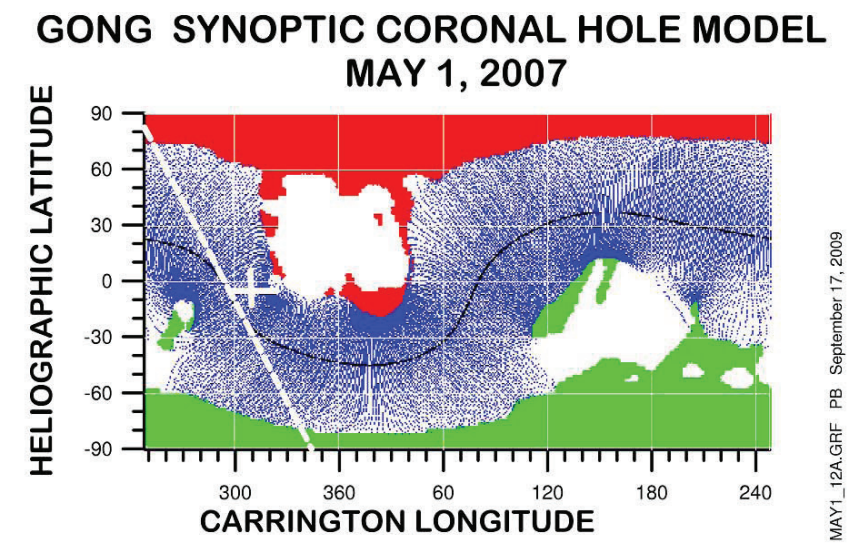

\section{GONG SYNOPTIC CORONAL HOLE MODEL MAY 13, 2007}

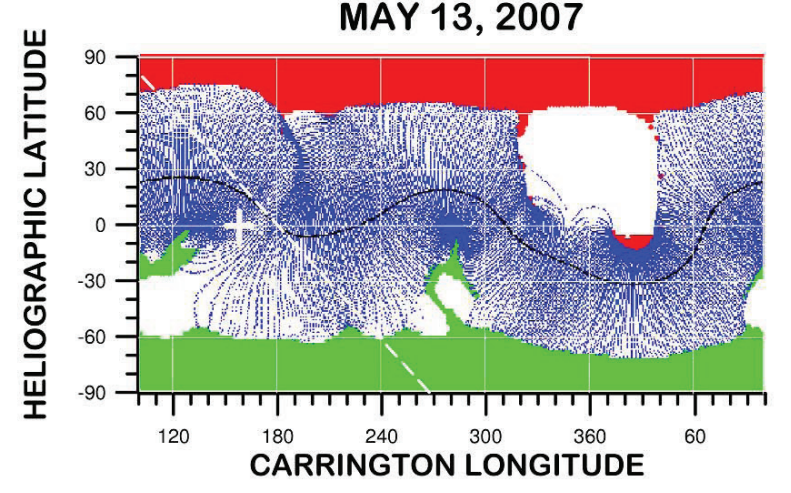

Fig. 4. Calculated coronal hole configurations according to GONG: Assuming a constant wind speed, we find the magnetic configuration at the source surface at the time, when a solar wind parcel left the Sun. Green denotes open field lines with positive polarity, red indicates negative polarity. The blue lines connect boundaries between open and closed magnetic field. The intercept of the black neutral line with the solar equator near the sub-terrestrial point (white cross) is taken for the location of the interface at the source. The parameter $d$, i.e., the slope of the interface at the source, has been derived from the white dashed line.

hence the subterrestrial longitude could also be taken as the sub-STEREO/A-longitude, given the large uncertainties of the backmapping procedure. Assuming that the solar wind speed had remained constant from the source surface to the site of observation and assuming the speeds we found in the low speed regimes, we find travel times of 5-6 days. With this information we traced the corresponding pictures of the source surface fields as shown in Fig. 4. The slope of the neutral line (drawn in black) closest to the subterrestrial point (white cross) is taken for the parameter $d$. For the first CIR of 7/8 May the determination was unambiguous, because the neutral line was nearly straight, when it crossed the solar equator, and a slope of $d=-1.7$ is derived for the white line in Fig. 4. Note that unlike in the case considered in Fig. 1

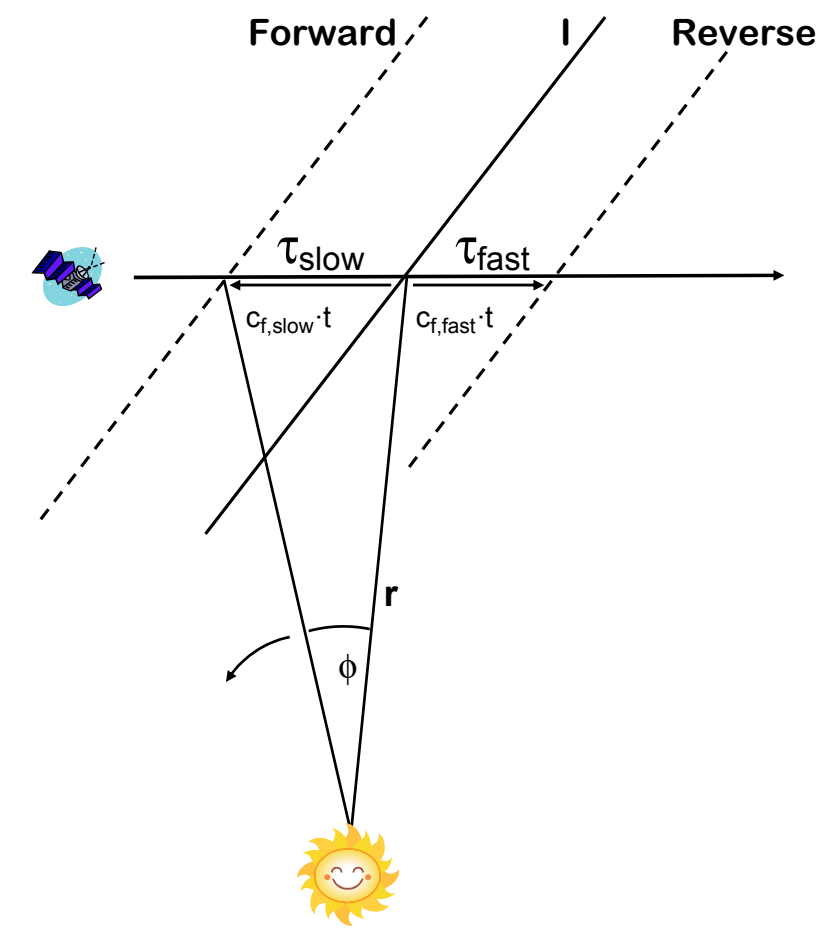

Fig. 5. Simplified schematic diagram for the estimation of time delays between crossings of reverse shocks (pressure waves), interface, and forward shocks (pressure waves).

of Lee (2000) the orientation of the interface at the source is different, since in both cases studied in this paper, the fast wind pushed from the Southern Hemisphere onto the interface. The polarity of the magnetic field in the high-speed streams measured at STEREO/A confirms the origin of the streams. The numerical value derived from Fig. 4 is in almost perfect agreement with our determination with the correlation procedure. For the second CIR of 18/19 May the determination is less straightforward because the neutral line is curved, and the result depends strongly on the assumption on the latitude of the source. Our best guess yields a slope of $d=-1$, which is significantly different from both values estimated with the least squares procedure and the correlation method. Given the uncertainties discussed above, and given the fact that under those circumstances the warping of the interface might change significantly during the 5 days travel time, it is not so surprising that our methods of inference do not work properly. On the other hand, the complicated evolution of the interface might help to explain why the second CIR did not develop a shock on the way to the observer.

The exact calculation of the width of the compression zone, i.e., the distance between forward and reverse shocks and the interface, or translated into timelag observed by a spacecraft traveling through the different regimes is more tricky since, in principle, it needs an integration over the entire history of the interface from its origin close to the Sun out 
to the observer. A coarse estimate is obtained with the simple idea that pressure waves tend to build up from the very beginning at the solar surface and that they propagate with the local fast mode speed $c_{\mathrm{f}}=\sqrt{c_{\mathrm{s}}^{2}+v_{\mathrm{A}}^{2}}$. Without knowledge of the ambient fast speeds between the starting point and the observer, one might assume that this parameter remains unchanged and simply give the normal distance from the front to the interface at any point by $c_{\mathrm{f}} \cdot t$, where $t$ is the travel time of the interface from the Sun to the observer. The distance $c_{\mathrm{f}} \cdot t$ (see Fig. 5) then defines the angle $\phi=c_{\mathrm{f}} \cdot t / r$. The travel time is given by $t=r / v_{I}$, where $v_{I}$ is the speed of the interface in the frame of the observer. With these relations, one obtains an order-of-magnitude estimate

$\phi \approx \frac{c_{f}}{v_{I}}=\frac{1}{M_{f}}$.

For slow wind a typical fast-mode Mach number $M_{\mathrm{f}}$ is 10 , whereas we assume about half this value for the fast wind. Thus we estimate the time intervals between the interface and the forward shock (pressure wave)

$\tau_{\text {slow }} \approx \frac{1}{\Omega_{\odot} \cdot M_{\text {f,slow }}} \approx 10 \mathrm{~h}$.

Similarly one finds a delay between the interface and the reverse shock (pressure wave):

$\tau_{\text {fast }} \approx \frac{1}{\Omega_{\odot} \cdot M_{\mathrm{f}, \text { fast }}} \approx 20 \mathrm{~h}$,

which is in rough agreement with the situation encountered in our second case (see Fig. 2).

Lee (2000) gives Eq. (46) to estimate the delays $\Delta \phi$ between fronts and interfaces

$\Delta \phi \simeq\left(V_{\mathrm{f}, \mathrm{j}} / V_{\mathrm{I}}\right)\left(\Omega r / V_{\mathrm{I}}\right)$,

where $V_{\mathrm{f}, \mathrm{j}}$ denotes the fast mode speeds in Region $\mathrm{j}$, i.e., in the undisturbed fast or slow wind regime, respectively on both sides of the interface. This expression might be used to put the delays on either side into relation to each other. As already seen from Eq. (5) the delays are essentially determined by the fast-mode speeds in the regimes adjacent to the interface. From Fig. 1, we note that in reality the delay in the fast regime is much longer than what would have been expected from these simple expressions. Generally the fastmode speed tends to decrease with increasing distance from the Sun. Hence, using the ambient fast-mode speeds at the site of the observer leads to an underestimation of the travel distances of the shock fronts from the interface. This effect might be somewhat more important in the fast wind regime, because of the steeper decrease of $c_{\mathrm{f}}$ due to the weaker bending of the Parker spirals. Improved estimates should include the entire history of the interface from the solar surface to the site of the observer. Future work might include comparisons of observations with the prediction of elaborated theoretical models such as the MHD model of the Center for Integrated Space Weather Modeling (CISM).

\section{Conclusions}

The current extended solar minimum provides excellent conditions to study the effects of CIRs in near-Earth-orbit environments. In the example of the CIR observed with STEREO/A on 7/8 May 2007, we have found that the simple planar approximation model of Lee (2000) provides a good description of the properties of the interplanetary medium preceding and following the interface. Although the local properties of the interface of the CIR of 18/19 May 2007, can be reasonably well parameterized with the model of Lee (2000), its evolution from the source surface to $1 \mathrm{AU}$ is apparently too complicated, to be described by a simple radial expansion of a locally planar interface. We find that the flow directions are the most sensitive markers for localizing the forward and reverse shocks, whereas changes in the absolute magnitudes of flow velocities are more difficult to identify unambiguously. Moreover, flow deflections can easily be detected in pressure waves even before an interface has developed shocks. The ambient fast mode speeds on both sides of the interface provide useful estimates of the time delays to be expected between forward shocks or pressure waves, the interface itself, and the reverse shocks or pressure waves. The PLASTIC instruments on STEREO provide an unprecedented opportunity to study the three-dimensional velocity distributions of iron ions with high statistical precision and excellent time resolution.

Acknowledgements. The authors thank all the individuals who designed and built the PLASTIC instruments for STEREO at the University of New Hampshire, the University of Bern, the Max-PlanckInstitute für extraterrestrische Physik and the University of Kiel, among them M. Boehm, J. Carroll, A. Crawshaw, M. Conti, P. Demaine, S. Ellis, J. A. Gaidos, J. Googins, M. Granoff, A. Gustafson, D. Heirtzler, B. King, U. Knauss, J. Levasseur, S. Longworth, K. Singer, S. Turco, P. Vachon, M. Vosbury, M. Widholm, J. Fischer, J. Jost, M. Sigrist, A. Etter, M. Ertl, E. Seidenschwang, and M. Koeten. We thank the topical editor, Robert Forsyth, for helpful discussions, and we acknowledge support by NASA STEREO contract NAS5-00132.

Topical Editor R. Forsyth thanks two anonymous referees for their help in evaluating this paper.

\section{References}

Aellig, M. R., Grünwaldt, H., Bochsler, P., Wurz, P., Hefti, S., Kallenbach, R., Ipavich, F. M., Hovestadt, D., Hilchenbach, M., and the CELIAS Team: Solar wind iron charge states observed with high time resolution with SOHO/CELIAS/CTOF, Proceedings of the Fifth SOHO Workshop, The corona and the solar wind near minimum activity, Oslo, Norway, ESA SP-404, pp. 157$161,1997$.

Aellig, M. R., Grünwaldt, H., Bochsler, P., Wurz, P., Hefti, S., Kallenbach, R., Ipavich, F. M., Axford, W. I., Balsiger, H., Bürgi, A., Coplan, M. A., Galvin, A. B., Geiss, J., Gliem, F., Gloeckler, G., Hilchenbach, M., Hovestadt, D., Hsieh, K. C., Klecker, B., Lee, M. A., Livi, S., Managadze, G. G., Marsch, E., 
Möbius, E., Neugebauer, M., Reiche, K.-U., Scholer, M., Verigin, M. I., and Wilken, B.: Iron freeze-in temperatures measured by SOHO/CELIAS/CTOF, J. Geophys. Res., 103, 17215-17222, 1998.

Burlaga, L. F.: Interplanetary magnetohydrodynamics, International Series on Astronomy and Astrophysics, Oxford University Press, New York, Oxford, 1995.

Carovillano, R. L. and Siscoe, G. L.: Corotating structure in the solar wind, Solar Phys. 8, 401-414, 1969.

Forsyth, R. J. and Marsch, E.: Solar origin and interplanetary evolution of stream interfaces, Space Sci. Rev., 89, 7-20, 1999.

Galvin, A. B., Kistler, L. M., Popecki, M. A., Farrugia, C. J., Simunac, K. D. C., Ellis, L., Möbius, E., Lee, M. A., Boehm, M., Carroll, J., Crashaw, A., Conti, M., Demaine, P., Ellis, S., Gaidos, J. A., Googins, J., Granoff, M., Gustafson, A., Heirtzler, D., King, B., Knauss, U., Levasseur, J., Longworth, S., Singer, K., Turco, S., Vachon, P., Vosbury, M., Widholm, M., Blush, L. M., Karrer, R., Bochsler, P., Daoudi, H., Etter, A., Fischer, J., Jost, J., Opitz, A., Sigrist, M., Wurz, P., Klecker, B., Ertl, M., Seidenschwang, E., Wimmer-Schweingruber, R. F., Koeten, M., Thompson, B., and Steinfeld, D.: The Plasma and Suprathermal Ion Composition (PLASTIC) Investigation on the STEREO Observatories, Space Sci. Rev., 136, 437-486, doi:10.1007/s11214007-9296-x, 2008.
Lee, M. A.: An analytical theory of the morphology, flows, and shock compressions at corotating interaction regions in the solar wind, J. Geophys. Res., 105, 10491-10500, 2000.

Hundhausen, A. J.: Nonlinear model of high-speed solar wind streams, J. Geophys. Res., 78, 1528-1542, 1973.

Karrer, R.: Ion optical calibration of the PLASTIC sensor on STEREO, PhD Thesis, University of Bern, 2007.

Neugebauer, M. and Snyder, C. W.: Mariner 2 observations of the solar wind - I. Average properties, J. Geophys. Res., 71, 44694484, 1966.

Pizzo, V. J.: A three-dimensional model of corotating streams in the solar wind. 1. Theoretical foundations, J. Geophys. Res., 83, 5563-5572, 1978.

Pizzo, V. J.: The evolution of corotating stream fronts near the ecliptic plane in the inner solar system. 2. Three-dimensional tilteddipole fronts, J. Geophys. Res., 96, 5405-5420, 1991.

Wimmer-Schweingruber, R. F., von Steiger, R., and Pärli, R.: Solar wind stream interfaces in corotating interaction regions: SWICS/Ulysses results, J. Geophys. Res., 102, 17407-17417, 1997. 